hep-th/0103090, PUPT-1979

November 1, 2018

\title{
Nonlocal Field Theories and their Gravity Duals
}

Aaron Bergman*, Keshav Dasgupta ${ }^{\star}$, Ori J. Ganor*, Joanna L. Karczmarek* and Govindan Rajesh ${ }^{\star}$

* Department of Physics, Jadwin Hall

Princeton University

NJ 08544, USA

$\diamond$ School of Natural Sciences

Institute of Advanced Study

Princeton, NJ 08540, USA

abergman, joannak@princeton. edu

origa@viper .princeton. edu

keshav, rajesh@sns.ias. edu

\begin{abstract}
The gravity duals of nonlocal field theories in the large $N$ limit exhibit a novel behavior near the boundary. To explore this, we present and study the duals of dipole theories - a particular class of nonlocal theories with fundamental dipole fields. The nonlocal interactions are manifest in the metric of the gravity dual and type-0 string theories make a surprising appearance. We compare the situation to that in noncommutative SYM.
\end{abstract}




\section{Introduction}

At the boundary, the metric of $\mathbf{A d S}_{d+1}, d s^{2}=\frac{\alpha^{\prime}}{u^{2}}\left(d t^{2}-d x_{1}^{2}-\cdots-d x_{d-1}^{2}-d u^{2}\right)$, becomes infinite. This way the boundary is described by classical geometry, and quantum gravity on $\mathbf{A d S}_{d+1}$ can correspond to a local field theory on a classical space [1, 2, 3, 4].

The AdS/CFT correspondence can be extended to field theories on a noncommutative space [5, 6, 7]. The gravity dual for the large $N$ limit of $\mathcal{N}=4$ Super-Yang-Mills theory on a noncommutative $\mathbb{R}^{4}$ (NCSYM) with the noncommutativity in the 2,3 directions has the metric [5, 6]

$$
d s^{2}=\frac{\alpha^{\prime}}{u^{2}}\left(d t^{2}-d x_{1}^{2}-h\left(d x_{2}^{2}+d x_{3}^{2}\right)-d u^{2}\right)
$$

where $h=\frac{u^{4}}{u^{4}+\theta^{2}}$ with $\theta=\theta_{23}$ the typical length scale in the theory. Here the boundary, $u \rightarrow 0$, is no longer classical. Indeed some components of the metric tend to zero on the boundary.

Our motivation for this paper is to understand how nonlocality in the field theory affects the metric of its gravity dual near the boundary. Unfortunately, field theories on noncommutative spaces can be quite complicated; they exhibit UV/IR mixing and nonlocal behavior on varying scales. UV/IR mixing, which means that high momentum is associated with largescale nonlocality and arbitrarily small momentum introduces a new short-distance scale, can even obstruct the renormalization procedure [8, 9]. Although $\mathcal{N}=4$ NCSYM is a finite theory and renormalizability is not an issue, noncommutative geometry doesn't appear to be the simplest way to introduce nonlocality. There is a simpler way.

We will study a class of nonlocal gauge field theories in which some of the fields correspond to dipoles of a constant length. Such theories were discussed in 10 in the context of Tduality in noncommutative geometry. They were realized in string theory in a different setting in [11]. Also see [12, 13, 14 for previous appearances of such a theory.

At low energies these "dipole theories" can be described as a deformation of $\mathcal{N}=4 \mathrm{SYM}$ by a vector operator of conformal dimension 5 . This can be compared to the deformation by a tensor operator of conformal dimension 6 that describes NCSYM at low energy [15, 16, 17]. If the conformal dimension and the size of the Lorentz representation is an indication of simplicity, then it is reasonable to expect that dipole theories might be simpler than NCSYM.

The more interesting questions, however, hover in the UV region of the theory. At 
distances shorter than the scale of the nonlocality, we expect to find new phenomena.

Our ultimate goal is to answer the following questions:

- How is the nonlocality of the dipole theory manifested in the boundary metric?

- How does this manifestation of nonlocality compare to that of noncommutative geometry? Are these features generic to the gravity duals of nonlocal field theories?

In section (7) we answer the first question for a particularly simple dipole theory. In the discussion, we show that this effect is analogous to a feature of the supergravity dual of noncommutative geometry. We also make some comments about the nature of the supergravity dual for generic nonlocal theories.

The particular dipole theory that we study breaks supersymmetry entirely. We chose to work with it because the supergravity equations are simplified. The fermionic degrees of freedom, however, require extra care. As we will argue, type-0 string theory with a strong RR field strength has to be used in order to correctly describe the gravity dual.

The paper is organized as follows. In section (2) we review the construction of dipole theories. In section (3) we describe a simple string theory realization of these theories and then calculate their gravity dual in section (4). In section (5) we compare the gravity dual and the field theory in the infrared. In section (6) we study the geometry of the gravity dual. In section (7) we demonstrate the nonlocality of the boundary. In section (8) we discuss a puzzle related to the nonlocal behavior of the fermions and argue that type-0 string theory has to be invoked to resolve it. In section (9) we compute some correlation functions and show how they exhibit some generic features of nonlocality. Finally, in section (10) we discuss how the features we have found here might be generic to the supergravity duals of all nonlocal field theories.

\section{Dipole Theories}

Dipole theories are nonlocal field theories that also break Lorentz invariance. They were obtained in [10] by studying the T-duals of twisted fields in noncommutative gauge theory. Below, we will describe how to make a dipole theory out of an ordinary field theory. 


\subsection{Definition}

We start with a local and Lorentz invariant field theory in $d$ dimensions. In order to turn it into a nonlocal theory we assign to every field $\Phi_{a}$ a vector $L_{a}^{\mu}(\mu=1 \ldots d)$. We will call this the "dipole vector" of the field.

The fields $\Phi_{a}$ can be scalars, fermions, or have higher spin. Next, we define a noncommutative product

$$
\left(\Phi_{1} \widetilde{\star} \Phi_{2}\right)_{x} \equiv \Phi_{1}\left(x-\frac{1}{2} L_{2}\right) \Phi_{2}\left(x+\frac{1}{2} L_{1}\right) .
$$

It is easy to check that this defines an associative product provided that the vector assignment is additive, that is, $\Phi_{1} \widetilde{\star} \Phi_{2}$ is assigned the dipole vector $L_{1}+L_{2}$. For CPT symmetry, we will require that if $\Phi$ has dipole vector $L$ then the charge conjugate field, $\Phi^{\dagger}$, is assigned the dipole vector $-L$. We will also require that gauge fields have zero dipole length.

In order to construct the Lagrangian of the dipole theory we need to replace the ordinary product of fields with the noncommutative $\widetilde{\star}$-product (11). In general, there might be some ordering ambiguity, but the theories we will consider below are $S U(N)$ gauge theories and have a natural ordering induced from the noncommutative products of $N \times N$ matrices.

We have seen that the requirement of associativity translates into a requirement of additivity for the dipole vectors. One way to ensure this is to have a global conserved charge in the theory such that a field $\Phi_{a}$ has charge $Q_{a}$. We then pick a constant vector $L^{\mu}$ and assign to every field $\Phi_{a}(a=1 \ldots n$, where $n$ is the number of fields in the theory) the dipole vector $Q_{a} L^{\mu}$. More generally, we can have $m$ global charges such that a field $\Phi_{a}$ has the charges $Q_{j a}(j=1 \ldots m)$. We can then pick a constant $d \times m$ matrix $\Theta^{\mu j}(\mu=1 \ldots d$ and $j=1 \ldots m)$ and assign the field $\Phi_{a}$ a dipole vector $\sum_{j=1}^{m} \Theta^{\mu j} Q_{j a}$.

Extending this definition by allowing $Q_{a}$ to be the momentum we see that noncommutative Yang-Mills theory can also be thought of as a dipole theory. The matrix $\Theta^{\mu j}$ then becomes $\Theta^{\mu \nu}(\nu=1 \ldots d)$ and is required to be antisymmetric. The dipole lengths are then both proportional to and transverse to the momentum [18, 19, 20].

\subsection{A Dipole Deformation of $\mathcal{N}=4$ SYM}

The dipole theories that we study in the rest of this paper can be obtained from ordinary $S U(N) \mathcal{N}=4 \mathrm{SYM}$ in $3+1 \mathrm{D}$ by turning the scalars and fermions into dipole fields. $\mathcal{N}=4$ 
SYM has 6 real scalars in the representation 6 of the R-symmetry group $S U(4)$ and 4 Weyl fermions in the representation 4 of $S U(4)$. We will use the global R-symmetry charges to determine the dipole vectors of the various fields as follows. Pick 3 constant commuting elements $V^{\mu} \in s u(4)(\mu=1 \ldots 3$ and we will not consider time-like dipole vectors in this paper), where $s u(4)$ is the Lie algebra of $S U(4)$. Take $V^{\mu}$ to have dimensions of length. Denote the matrix elements of $V^{\mu}$ in the representation 4 as $\hat{U}_{j \bar{k}}^{\mu}(j, \bar{k}=1 \ldots 4)$. Here $\hat{U}^{\mu}$ is a traceless Hermitian $4 \times 4$ matrix. Denote the matrix elements of $V^{\mu}$ in the representation 6 as $M_{a b}^{\mu}(a, b=1 \ldots 6)$. $M^{\mu}$ is a real antisymmetric $6 \times 6$ matrix.

Let $u_{a}^{(l)}(a, l=1 \ldots 6)$ be an eigenvector of $M^{\mu}$ with (real) eigenvalue $\tilde{L}_{l}^{\mu}$ so that $\sum_{b} M_{a b}^{\mu} u_{b}^{(l)}=\tilde{L}_{l}^{\mu} u_{a}^{(l)} \cdot u_{a}^{(l)}$ does not depend on $\mu$ because $\left[M^{\mu}, M^{\nu}\right]=0$. Let $\phi_{a}(a=1 \ldots 6)$ be the 6 real scalar fields of $\mathcal{N}=4$ SYM. Then the complex valued scalar fields $\phi^{(l)} \equiv \sum_{a} u_{a}^{(l)} \phi_{a}$ are assigned a dipole vector with components $2 \pi \tilde{L}_{l}^{\mu}(\mu=1 \ldots d)$. Similarly, the fermionic fields are assigned dipole vectors that are determined by the eigenvalues of the matrices $\hat{U}^{\mu}$.

\subsection{Supersymmetry}

The dipole theories obtained from $\mathcal{N}=4$ SYM in the previous subsection are parameterized by $d$ constant traceless Hermitian $4 \times 4$ matrices $\hat{U}^{\mu}$. For simplicity we will set $\hat{U}^{1}=\hat{U}^{2}=0$ and $\hat{U} \equiv \hat{U}^{3}$. Thus, the dipole vectors are all in the $3^{\text {rd }}$ direction. The matrix $\hat{U}$ has dimensions of length, and its eigenvalues determine the dipole vectors of the various fields. Let the eigenvalues be $\alpha_{1}, \alpha_{2}, \alpha_{3},-\left(\alpha_{1}+\alpha_{2}+\alpha_{3}\right)$. Then, the dipole vectors of the various scalar fields are given by $\pm\left(\alpha_{i}+\alpha_{j}\right)(1 \leq i<j \leq 3)$.

The number of supersymmetries that are preserved by the dipole theory is determined by the rank $r$ of $\hat{U}$ :

- If $r=4$, then the theory is not supersymmetric at all.

- If $r=3$, there is one zero eigenvalue that we take by convention to be $\alpha_{3}=0$, and the theory has $\mathcal{N}=1$ supersymmetry.

- If $r=2$, there are two zero eigenvalues that we take to be $\alpha_{2}=\alpha_{3}=0$. The theory then has $\mathcal{N}=2$ supersymmetry. The vector multiplet of $\mathcal{N}=4 \mathrm{SYM}$ decomposes as a vector multiplet and a hypermultiplet of $\mathcal{N}=2 \mathrm{SYM}$. All the fields in the $\mathcal{N}=2$ vector multiplet have dipole vector 0 , and the fields in the hypermultiplet have dipole vectors 
$\pm \alpha_{1}$.

Because we can realize dipole theories without supersymmetry, one might ask if poles similar to those discovered in [8, 9] might arise in the perturbative expansion of the theory. In fact, they do not. This can be seen by examining the expression of [8, 9] for the effective cutoff

$$
\Lambda_{\text {eff }} \rightarrow \frac{1}{\sqrt{\Lambda^{-2}+(\theta p)^{2}}} .
$$

We recognize $\theta p$ as the length of the dipoles in noncommutative geometry. Thus, the analogous expression in our theory is

$$
\Lambda_{\text {eff }} \rightarrow \frac{1}{\sqrt{\Lambda^{-2}+L^{2}}}
$$

which, as it is independent of the momenta, gives rise to no new poles.

\section{String Theory Realization of Dipole Theories}

In order to find the gravity dual of the large $N$ limit of a particular dipole theory, we need to find a simple string theory realization for it. We now do this for a large class of dipole theories.

In [11], a realization of dipole theories with $\mathcal{N}=2$ supersymmetry was suggested using D3-branes that probe the center of a modified Taub-NUT geometry. While this realization is convenient for a BPS analysis it is hard to extract the gravity dual from it, and it is not obvious how to generalize it to dipole theories that break $\mathcal{N}=2$ supersymmetry.

Fortunately, the Taub-NUT space that was used in [11] is not an essential ingredient. We can find an alternative setting that has the same behavior near the brane probes. This setting, which we will describe below, has the disadvantage that the geometry is not asymptotically Euclidean at infinity. Nevertheless, it has been constructed in string theory [21] and is good for extracting the gravity duals that we seek. Other worldsheet CFTs that break Lorentz invariance have been studied in [22].

The backgrounds that we consider are twisted versions of type-II string theory. They are related to the Melvin solution [23] and are in fact identical to the backgrounds discussed in [24] and more recently in [25, 26]. As was shown in [24, the twisted backgrounds are unstable, 
and the instability is similar to that discussed in [27]. This instability is exponentially suppressed as $g_{s} \rightarrow 0$ and is likely to be completely absent when some supersymmetry is preserved. For the time being we will ignore the instability. We will return to this point in the discussion.

\subsection{The T-dual of a Twist}

We will first describe a type-II background without branes and then later we will add the brane probes. Consider type-IIA string theory on a space that is $\mathbb{R}^{9,1}$ modded out by the isometry

$$
\mathcal{U}:\left(x_{0}, x_{1}, x_{2}, x_{3},\left\{x_{3+a}\right\}_{a=1}^{6}\right) \mapsto\left(x_{0}, x_{1}, x_{2}, x_{3}+2 \pi R_{3},\left\{\sum_{b=1}^{6} O_{b a} x_{3+a}\right\}_{a=1}^{6}\right) .
$$

Here $O \in S O(6)$ is an orthogonal matrix. The twisted compactification is parameterized by $R_{3}$ and, because we need to define the action on fermions, an element of $\operatorname{Spin}(6) \cong S U(4)$. This background is, in general, modified by quantum corrections, but $O$ and $R_{3}$ are defined by their asymptotic values at infinity. We will denote this background by $X\left(O, R_{3}\right)$. Note that if $R_{3}>0$ the isometry $\mathcal{U}$ has no fixed points and therefore $O$ is not necessarily of finite order.

Now consider probing $X\left(O, R_{3}\right)$ with D2-branes in directions $\left(x_{0}, x_{1}, x_{2}\right)$ and then taking

the limit $R_{3} \rightarrow 0$ together with $O=e^{\frac{2 \pi i R_{3} M}{\alpha^{\prime}}}$ where $M$ is a finite matrix of the Lie algebra $s o(6) \cong s u(4)$ with dimensions of length and $\frac{\alpha^{\prime}}{2 \pi}$ is the inverse string tension.

When $M=0$, we can perform T-duality to transform the D2-branes into D3-branes. When $M \neq 0$, we will now show the low energy description of the probe is a dipole theory.

\subsection{Branes Probing Dual Twists}

We wish to find the low energy Lagrangian describing D2-branes that probe the twisted geometry of subsection (3.1). The light degrees of freedom come from the strings with two Dirichlet boundary conditions, i.e., fundamental strings with ends on the D2-branes. Because $R_{3} \rightarrow 0$, we have to set the string oscillators to their ground states, but the winding number can be arbitrary. 
To obtain the Lagrangian, we can adopt a procedure similar to the one described in [28, 29, 30] for noncommutative gauge theories. Also, the construction that we present here is reminiscent of the construction in [31]. In momentum space, the action of the dipole theory is obtained from the action of $\mathcal{N}=4 \mathrm{SYM}$ by inserting certain phases. Let $\Phi_{1}\left(p_{1}\right), \ldots, \Phi_{n}\left(p_{n}\right)$ be fields in the adjoint representation of $U(N)$ and suppose that $\mathcal{N}=4 \mathrm{SYM}$ has a term of the form

$$
\operatorname{tr}\left\{\Phi_{1}\left(p_{1}\right) \cdots \Phi_{n}\left(p_{n}\right)\right\}
$$

in the Lagrangian (of course $n \leq 4$ ). The variables $p_{i}$ are the momenta. Let the dipole vectors of the fields be $L_{1}, \ldots, L_{n}$. We have

$$
\sum_{i=1}^{n} L_{i}=0, \quad \sum_{i=1}^{n} p_{i}=0 .
$$

The dipole theory is obtained from the ordinary $\mathcal{N}=4 \mathrm{SYM}$ theory by inserting the phases

$$
e^{i \sum_{1 \leq i<j \leq n} p_{i} L_{j}}
$$

in front of terms like (2). Now let us consider branes probing $X\left(O, R_{3}\right)$. For simplicity, let us assume that the twist, $O$, acts only on $Z \equiv X^{8}+i X^{9}$ as $Z \rightarrow e^{i \alpha} Z$. We will refer to the angular momentum corresponding to rotation in the $Z$-plane as the $Z$-charge.

In the case that $\alpha=0$ we know that the theory on the D2-brane probe is $\mathcal{N}=4 \mathrm{SYM}$. The states with momentum along the $3^{\text {rd }}$ direction, in the SYM theory, correspond to winding states along the $3^{\text {rd }}$ direction in the string theory setting.

Now let us turn on the twist, $\alpha$. Consider a string disc amplitude that calculates the interaction of $n$ open string states with winding numbers $w_{1}, \ldots, w_{n}$ and with $Z$-charges $q_{1}, \ldots, q_{n}$. The worldsheet theory has a global $U(1)$ symmetry corresponding to the $Z$ charge. The string vertex operators that correspond to the external states are charged under this $U(1)$ symmetry. The disc worldsheet has cuts that emanate from the external vertex operators on the boundary. Along the $j^{t h}$ cut, the worldsheet field $Z$ jumps by a phase $e^{i \alpha q_{j}}$. We can redefine the field $Z$ to be continuous, but then there will be additional phases coming from the vertex operators on the boundary. It is easy to see that this phase is

$$
e^{i \alpha \sum_{1 \leq i<j \leq n} w_{i} q_{j}}
$$

This is illustrated in figure 1. It agrees with (3) because

$$
L_{j}=\alpha^{\prime} \frac{\alpha}{R_{3}} q_{j}, \quad p_{j}=\alpha^{\prime-1} R_{3} w_{j} .
$$




\section{Upper Half Plane}

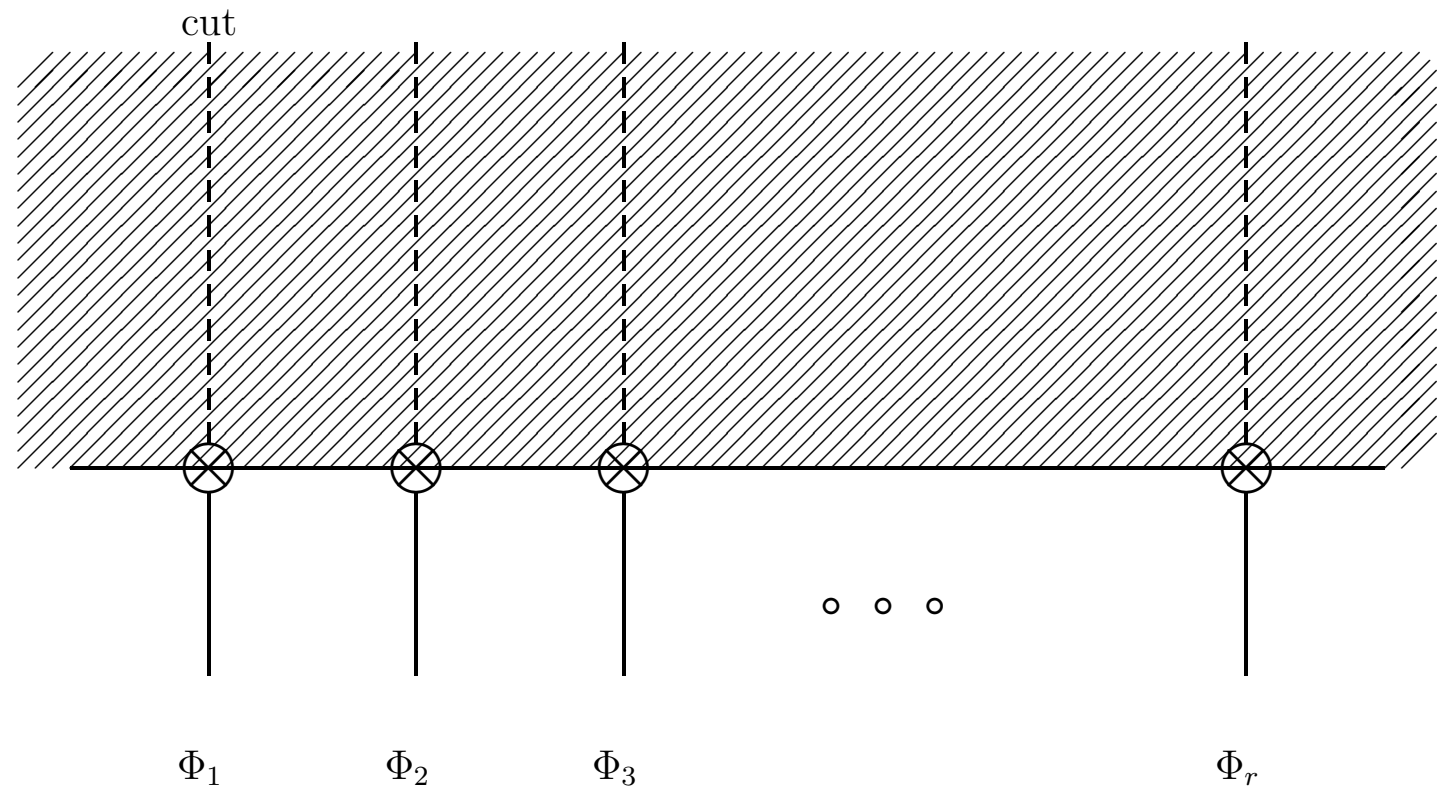

Figure 1: An $r$-point amplitude with vertex operators that carry winding numbers. It requires $r$ cuts on the worldsheet.

In $3+1 \mathrm{D}$, the photon of the $U(1) \subset U(N)$ center of the gauge group is likely to become massive via a dynamical mechanism similar to the one described in [32] for quiver theories, so that the gauge group is actually just $S U(N)$, but we will ignore this for the time being.

\section{Supergravity Solution for a Twisted Brane}

We now turn to the task of describing the supergravity duals of these dipole theories. We will use the string theory realization of dipole theories as described in the previous section.

We will find the exact classical supergravity solutions in four steps:

1. We start with the D3-brane solution of type-IIB classical supergravity and compactify one of the directions parallel to the D3-branes. We will call it the $3^{\text {rd }}$ direction.

2. We perform T-duality on the $3^{\text {rd }}$ direction to obtain a solution that describes D2branes in type-IIA. The solution, however, will be translationally invariant along the $3^{\text {rd }}$ direction, and, as such, it describes smeared rather than localized D2-branes.

3. We now insert a transverse $S O(6)$ twist into the geometry by hand. This is accomplished 
by simply changing the boundary conditions for the 6 transverse coordinates as we complete a circle around the $3^{\text {rd }}$ direction. Locally, the metric is unchanged.

4. Finally, we use T-duality to turn the smeared D2-branes back to D3-branes.

In this paper we will restrict ourselves to cases where all the dipole vectors of the fields in the theory are oriented in the same direction. This was direction 3 above. In appendix (A) we present the generalization for generic dipole vectors. We now turn to the details.

\subsection{The Type-IIB D3-Brane}

First, the conventions. We work in the $(+,-, \ldots,-)$ metric. Greek indices are $\mu, \nu=0 \ldots 2$. The time direction is $t=x_{0}$. The direction that we T-dualize is the $3^{\text {rd }}$. The remaining directions, perpendicular to the brane, are labeled by roman indices $a, b=4 \ldots 9$. All metrics will be in string frame.

We start with the metric for a D3-brane (note that all the $x$ 's have dimensions of length)

$$
d s_{\mathrm{str}}^{2}=H^{-\frac{1}{2}}\left(d t^{2}-d x_{1}^{2}-d x_{2}^{2}-d x_{3}^{2}\right)-H^{\frac{1}{2}}\left(\delta_{a b} d x^{a} d x^{b}\right)
$$

where

$$
H=1+\frac{\mathcal{R}^{4}}{r^{4}}, \quad \mathcal{R}^{4}=4 \pi g_{s} N \alpha^{2}, \quad r^{2}=\delta_{a b} x^{a} x^{b},
$$

and we have the following backgrounds for the RR 4-from potential and the dilaton respectively

$$
C_{0123}^{(4)}=-H^{-1}, \quad e^{2 \phi}=e^{2 \phi_{0}}
$$

Next, we compactify along $x^{3}$ with radius $R_{3} \equiv R$. The metric is now

$$
d s^{2}=H^{-\frac{1}{2}}\left(d t^{2}-\delta_{\mu \nu} d x^{\mu} d x^{\nu}-R^{2} d \hat{x}_{3}^{2}\right)-H^{\frac{1}{2}}\left(\delta_{a b} d x^{a} d x^{b}\right)
$$

Note that $\hat{x}_{3}$ is now dimensionless and periodic $\hat{x}_{3} \sim \hat{x}_{3}+2 \pi$.

\subsection{A Smeared D2-Brane with a Twist}

We now T-dualize around $x_{3}$. Following [33, 34, 35], we have

$$
\begin{gathered}
C_{012}^{(3)}=\frac{8}{3} H^{-1} \quad e^{2\left(\phi-\phi_{0}\right)}=\frac{\alpha^{\prime}}{R^{2}} H^{\frac{1}{2}} \\
d s^{2}=H^{-\frac{1}{2}}\left(d t^{2}-d x_{1}^{2}-d x_{2}^{2}\right)-H^{\frac{1}{2}}\left(\frac{\alpha^{\prime 2}}{R^{2}} d \hat{x}_{3}^{2}+d x_{4}^{2}+\cdots+d x_{9}^{2}\right)
\end{gathered}
$$


This is a smeared D2-brane. We can now add a twist to the transverse directions $x_{4}, \cdots, x_{9}$ as we travel around the circle $x_{3}$. In particular, we take an element of the Lie algebra $s o(6)$, $\Omega_{a b}$, and change the metric to

$$
d s^{2}=H^{-\frac{1}{2}}\left(d t^{2}-d x_{1}^{2}-d x_{2}^{2}\right)-H^{\frac{1}{2}}\left\{\alpha^{\prime 2} R^{-2} d \hat{x}_{3}^{2}+\sum_{a}\left(d x_{a}-\sum_{b} \Omega_{a b} x_{b} d \hat{x}_{3}\right)^{2}\right\}
$$

We can expand this out, giving

$$
\begin{aligned}
d s^{2}= & H^{-\frac{1}{2}}\left(d t^{2}-d x_{1}^{2}-d x_{2}^{2}\right) \\
& -H^{\frac{1}{2}}\left\{\left(\alpha^{\prime 2} R^{-2}+\vec{x}^{\top} \Omega^{\top} \Omega \vec{x}\right) d \hat{x}_{3}^{2}+d \vec{x}^{\top} d \vec{x}-2 d \vec{x}^{\top} \Omega \vec{x} d \hat{x}_{3}\right\}
\end{aligned}
$$

where $\vec{x}$ is the vector formed by $x_{a}(a=4 \ldots 9)$.

\subsection{And Back to the D3-Brane}

Once again, we apply the T-duality formulae (recall that $\hat{x}_{3}$ is dimensionless, while all the other coordinates have dimension of length)

$$
\begin{aligned}
d s^{2}= & H^{-\frac{1}{2}}\left(d t^{2}-d x_{1}^{2}-d x_{2}^{2}-\frac{\alpha^{\prime 2}}{\alpha^{\prime 2} R^{-2}+\vec{x}^{\top} \Omega^{\top} \Omega \vec{x}} d \hat{x}_{3}^{2}\right) \\
& -H^{\frac{1}{2}}\left(d \vec{x}^{\top} d \vec{x}-\frac{\left(d \vec{x}^{\top} \Omega \vec{x}\right)^{2}}{\alpha^{\prime 2} R^{-2}+\vec{x}^{\top} \Omega^{\top} \Omega \vec{x}}\right)
\end{aligned}
$$

We also have

$$
\begin{aligned}
C_{3012}^{(4)} & =H^{-1} \\
\sum_{a} B_{\hat{3} a} d x^{a} & =-\frac{d \vec{x}^{\top} \Omega \vec{x}}{\alpha^{\prime 2} R^{-2}+\vec{x}^{\top} \Omega^{\top} \Omega \vec{x}} \\
e^{2\left(\phi-\phi_{0}\right)} & =\frac{1}{1+\alpha^{\prime-2} R^{2} \vec{x}^{\top} \Omega^{\top} \Omega \vec{x}}
\end{aligned}
$$

which we will address later on.

Defining $\vec{x}=r \hat{n}$ so that $\|\hat{n}\|=1$, our metric becomes

$$
\begin{aligned}
d s^{2}= & H^{-\frac{1}{2}}\left(d t^{2}-d x_{1}^{2}-d x_{2}^{2}-\frac{\alpha^{2}}{\alpha^{\prime 2} R^{-2}+r^{2} \hat{n}^{\top} \Omega^{\top} \Omega \hat{n}} d \hat{x}_{3}^{2}\right) \\
& -H^{\frac{1}{2}}\left(d r^{2}+r^{2} d \hat{n}^{\top} d \hat{n}-\frac{r^{4}}{\alpha^{\prime 2} R^{-2}+r^{2} \hat{n}^{\top} \Omega^{\top} \Omega \hat{n}}\left(\hat{n}^{\top} \Omega^{\top} d \hat{n}\right)^{2}\right)
\end{aligned}
$$




\subsection{The Near-Horizon Limit}

In these coordinates, the horizon is at $r=0$, and so the near-horizon limit is $r$ small. We can therefore approximate

$$
H^{\frac{1}{2}}=\sqrt{1+\frac{\mathcal{R}^{4}}{r^{4}}} \sim\left(\frac{\mathcal{R}}{r}\right)^{2} .
$$

Substituting this into the metric, we obtain

$$
\begin{aligned}
d s^{2}= & (r / \mathcal{R})^{2}\left(d t^{2}-d x_{1}^{2}-d x_{2}^{2}-\frac{\alpha^{\prime 2}}{\alpha^{\prime 2} R^{-2}+r^{2} \hat{n}^{\top} \Omega^{\top} \Omega \hat{n}} d \hat{x}_{3}^{2}\right) \\
& -\frac{\mathcal{R}^{2}}{r^{2}} d r^{2}-\mathcal{R}^{2}\left(d \hat{n}^{\top} d \hat{n}-\frac{1}{\alpha^{\prime 2}(r R)^{-2}+\hat{n}^{\top} \Omega^{\top} \Omega \hat{n}}\left(\hat{n}^{\top} \Omega^{\top} d \hat{n}\right)^{2}\right)
\end{aligned}
$$

Finally, we make the substitution $u=\mathcal{R}^{2} / r$

$$
\begin{aligned}
d s^{2}= & \frac{\mathcal{R}^{2}}{u^{2}}\left(d t^{2}-d x_{1}^{2}-d x_{2}^{2}-\frac{u^{2}}{\left(\frac{u}{R}\right)^{2}+\lambda^{2} \hat{n}^{\top} \Omega^{\top} \Omega \hat{n}} d \hat{x}_{3}^{2}\right)-\mathcal{R}^{2} \frac{d u^{2}}{u^{2}} \\
& -\mathcal{R}^{2}\left(d \hat{n}^{\top} d \hat{n}-\frac{\lambda^{2}}{\left(\frac{u}{R}\right)^{2}+\lambda^{2} \hat{n}^{\top} \Omega^{\top} \Omega \hat{n}}\left(\hat{n}^{\top} \Omega^{\top} d \hat{n}\right)^{2}\right)
\end{aligned}
$$

where $\lambda^{2} \equiv \frac{\mathcal{R}^{4}}{\alpha^{\prime 2}}=4 \pi g_{\mathrm{YM}}^{2} N$. The dilaton and the NSNS 2-form field are

$$
\begin{aligned}
\sum_{a} B_{\hat{3} a} d \hat{n}^{a} & =-\frac{\lambda^{2}}{\frac{u^{2}}{R^{2}}+\lambda^{2} \hat{n}^{\top} \Omega^{\top} \Omega \hat{n}} d \hat{n}^{\top} \Omega \hat{n}, \\
e^{2\left(\phi-\phi_{0}\right)} & =\frac{1}{1+\frac{R^{2}}{u^{2}} \lambda^{2} \hat{n}^{\top} \Omega^{\top} \Omega \hat{n}}
\end{aligned}
$$

Now we take the limit $R \rightarrow \infty$ keeping $R \Omega=M$ fixed. We also redefine $\hat{x}_{3}=\frac{x_{3}}{R}$. Note that $x_{3}, u$ and $M$ have dimensions of length. We find

$$
\begin{aligned}
d s^{2}= & \frac{\mathcal{R}^{2}}{u^{2}}\left(d t^{2}-d x_{1}^{2}-d x_{2}^{2}-d u^{2}-\frac{u^{2}}{u^{2}+\lambda^{2} \hat{n}^{\top} M^{\top} M \hat{n}} d x_{3}^{2}\right) \\
& -\mathcal{R}^{2}\left(d \hat{n}^{\top} d \hat{n}-\frac{\lambda^{2}}{u^{2}+\lambda^{2} \hat{n}^{\top} M^{\top} M \hat{n}}\left(\hat{n}^{\top} M^{\top} d \hat{n}\right)^{2}\right)
\end{aligned}
$$

The NSNS 2-form field and the dilaton are

$$
\begin{aligned}
\sum_{a} B_{3 a} d \hat{n}^{a} & =-\frac{\lambda^{2}}{u^{2}+\lambda^{2} \hat{n}^{\top} M^{\top} M \hat{n}} d \hat{n}^{\top} M \hat{n}, \\
e^{2\left(\phi-\phi_{0}\right)} & =\frac{u^{2}}{u^{2}+\lambda^{2} \hat{n}^{\top} M^{\top} M \hat{n}}
\end{aligned}
$$

Given this form of the metric it is not obvious that the region $\frac{\mathcal{R}}{r} \gg 1$ indeed decouples from the bulk, as we have assumed. In principle, one can calculate scattering amplitudes for 
gravitons as in [36, 37]. In some cases one can see from the scattering amplitudes that the bulk does not decouple (see for instance [38]).

In our case, the geometry is strongly coupled when $u$ is small, as will be discussed in more detail in subsection (7.1), and evaluating the scattering amplitudes is difficult. Nevertheless, there is no reason to expect that the bulk will not decouple. The dipole-theories describe well defined renormalizable theories that do not require additional degrees of freedom in the UV.

\section{Comparison to the Field Theory in the Infrared}

For $M=0$, the metric (4) describes $\mathbf{A d S}_{5} \times \mathbf{S}^{5}$ with boundary at $u=0$. The IR region corresponds to large $u$. For large $u$ the deviation from the standard $\mathbf{A d S}_{5} \times \mathbf{S}^{5}$ metric describes a deformation of $\mathcal{N}=4$ SYM by irrelevant operators.

In [10], the first order correction to the SYM Lagrangian was determined as the dipole length times the dimension 5 operator

$$
\mathcal{O}_{\mu}^{I J}=\frac{i}{g_{\mathrm{YM}}^{2}} \operatorname{tr}\left\{F_{\mu}{ }^{\nu} \Phi^{[I} D_{\nu} \Phi^{J]}+\sum_{K}\left(D_{\mu} \Phi^{K}\right) \Phi^{[K} \Phi^{I} \Phi^{J]}\right\}+\text { fermions }
$$

Here $I, J=1 \ldots 6$ are R-symmetry indices, $\Phi^{I}(I=1 \ldots 6)$ are the scalars, $D_{\mu}=\partial_{\mu}-$ $i\left[A_{\mu}, \cdot\right]$ is the covariant derivative, $F_{\mu \nu}$ is the field strength and [...] means complete antisymmetrization. $\mathcal{O}_{\mu}^{I J}$ is a vector operator that transforms in the $\mathbf{1 5}$ of the R-symmetry group $S U(4)$.

We should be able to find the dual of this in the supergravity. The only vector field on AdS that we have obtained is the two-form

$$
\sum_{a} B_{3 a} d \hat{n}^{a}=-\frac{\lambda^{2}}{u^{2}+\lambda^{2} \hat{n}^{\top} M^{\top} M \hat{n}} d \hat{n}^{\top} M \hat{n}
$$

In particular, at $\mathcal{O}(L)$, we have

$$
B_{3 a}=-\frac{\lambda^{2}}{u^{2}} M_{a b} \hat{n}^{b}
$$

When acted upon by $\hat{O} \in S O(6), B_{3 a}$ transforms as

$$
\hat{O}(B)_{3 a}=-\frac{\lambda^{2}}{u^{2}} \hat{O}_{a b} M_{b c} \hat{O}_{c d}^{-1} \hat{n}^{d}
$$


Because $M$ is in the Lie algebra, so(6), we recognize this as the adjoint representation, $\mathbf{1 5}$ or, in terms of spherical harmonics, the $k=1, M^{2}=8$ representation in 39. This corresponds to a dimension 5 operator. Referring to the table of [40], this is a linear combination of $\delta^{3} \bar{\delta} \mathcal{O}_{3}$ and $\bar{\delta}^{3} \delta \mathcal{O}_{3}$ where $\mathcal{O}_{p}$ is the chiral primary $\operatorname{tr}\left\{\Phi^{\left(I_{1}\right.} \Phi^{I_{2}} \ldots \Phi^{\left.I_{p}\right)}\right\}$ - traces. Here, $\delta \mathcal{O}$ $(\bar{\delta} \mathcal{O})$ represents either the commutator or anti-commutator, as appropriate, of $\mathcal{O}$ with the supersymmetry generator $Q(\bar{Q})$. We also note from the table that this is a vector operator, as expected.

For large $u$, we can make the following expansion

$$
\frac{1}{u^{2}+\alpha^{2}}=\frac{1}{u^{2}}\left(1-\frac{\alpha^{2}}{u^{2}}+\frac{\alpha^{2}}{u^{4}}+\cdots\right) \text {. }
$$

Thus, we obtain deformations that are multiplied by a higher power of the dipole length. We will work through some of the $\mathcal{O}\left(L^{2}\right)$ terms.

For the deformation of the sphere $\mathbf{S}^{5}$, we have

$$
h_{(a b)}=-\frac{\mathcal{R}^{2} \lambda^{2} M_{a c} \hat{n}^{c} M_{b d} \hat{n}^{d}}{u^{2}}\left(1+\mathcal{O}\left(L^{2}\right)\right)-\text { trace }
$$

We immediately see from the above that this must transform in some component of the $\mathbf{1 5} \otimes_{\text {sym }} \mathbf{1 5}=\mathbf{8 4} \oplus \mathbf{2 0} \oplus \mathbf{1 5} \oplus \mathbf{1}$. With a little work, one can see that the correct component is the 84. In terms of spherical harmonics, this corresponds to the $k=1, M^{2}=12$ field in [39]. We can read off the weights from the Young tableaux, giving $(2,0,2)$. Following the table of [40], we can see that this corresponds to the operator $\delta^{2} \bar{\delta}^{2} \mathcal{O}_{4}$. This is a scalar operator of dimension 6 .

Also, at $\mathcal{O}\left(L^{2}\right)$, there are a number of other deformations arising from the term $\hat{n}^{\top} M^{\top} M \hat{n}$. Specifically, there are $h_{a}^{a}$, the dilaton and the 33 component of the metric on $\mathbf{A d S}_{5} \cdot \operatorname{As}^{\top} M$ is a symmetric $6 \times 6$ matrix, we can see that these transform in the $\mathbf{2 0} \oplus \mathbf{1}$. For the dilaton, we can identify the mass of the $\mathbf{2 0}$ as $M^{2}=12$. Thus, this is also a dimension 6 operator. Again, reading from [40] identifies the operator as some combination of $\delta^{4}$ and $\bar{\delta}^{4}$ acting on $\mathcal{O}_{4}$. For the trace of the metric, we are presented with a problem. The metric has a term in its equation of motion arising from the product of two three-form NSNS fluxes, giving a term also of order $\mathcal{O}\left(L^{2}\right)$. Thus, it can no longer be treated as a linear perturbation on the AdS background. The fact that we got the correct answer for the traceless part of the metric perturbation is due to the fact that the product of the NSNS fluxes does not have any component that transforms in the $\mathbf{8 4}$, and so it can be treated as a linear perturbation. 


\section{The Geometry of the Supergravity}

We now investigate some of the geometrical features of the metric (4). The key things to note are the behavior of $x_{3}$ coordinate and the $\mathbf{S}^{5}$ as a function of $u$. We first discuss the generic behavior and then give a detailed analysis of a useful special case that will occupy us for the remainder of the paper. General deformations of the $\mathbf{S}^{5}$ were also studied in a slightly different context in [41].

\subsection{The Boundary}

The behavior near the boundary is governed by the rank of $M$. For maximal rank, the quadratic function $\hat{n}^{\top} M^{\top} M \hat{n}$ is always positive definite. It has 12 local extrema on $\mathbf{S}^{5}$. These consist of pairs of antipodal points - each pair corresponds to an eigenvector of $M^{\top} M$ with the two $( \pm)$ sign options. The metric (田) is asymptotically $\mathbf{A d S}_{4} \times \mathbf{S}^{1} \times \mathbf{S}^{5}$ where the $\mathbf{S}^{5}$ is deformed, and both the $\mathbf{S}^{1}$ and $\mathbf{S}^{5}$ are small compared to the AdS.

If the rank of $M$ is less than maximal, the quadratic form $\hat{n}^{\top} M^{\top} M \hat{n}$ has a locus of zeroes. This locus is $\mathbf{S}^{r-1}$ where $r=2,4$ are the possible nonzero values for the rank. Locally on the zero locus, the metric is indistinguishable from ordinary $\mathbf{A} \mathbf{d} \mathbf{S}_{5} \times \mathbf{S}^{5}$. This should be related to the fact that some scalar fields do not have a dipole length. We do not claim to understand the exact connection.

The metric on $\mathbf{S}^{5}$ becomes degenerate as $u \rightarrow 0$. For $M$ of maximal rank, the metric on $\mathbf{S}^{5}$ at $u=0$ is

$$
d s^{2}=\mathcal{R}^{2} d \hat{n}^{\top} d \hat{n}-\frac{\mathcal{R}^{2}\left(\hat{n}^{\top} M^{\top} d \hat{n}\right)^{2}}{\hat{n}^{\top} M^{\top} M \hat{n}}
$$

Let $\hat{n}$, a unit vector in $\mathbb{R}^{6}$, parameterize a point $p \in \mathbf{S}^{5}$. Then $M \hat{n}$ defines a direction in the tangent space $T_{p} \mathbf{S}^{5}$, since $\hat{n}^{\top} M \hat{n}=0$. It is easy to see that the metric is degenerate along this direction. Thus, $M \hat{n}$ defines a vector field on $\mathbf{S}^{5}$ along which the metric is degenerate. This is the vector field induced by the infinitesimal $S O(6)$ action on $\mathbf{S}^{5}$ given by $M \in s o(6)$. To analyze the degenerate $\mathbf{S}^{5}$ further we need to know more about the eigenvalues of $M$. Let the eigenvalues be $\pm i \alpha_{1}, \pm i \alpha_{2}, \pm i \alpha_{3}$. If $\alpha_{1}=\alpha_{2}=\alpha_{3}$ then the flow lines of the vector field $M \hat{n}$ are closed circles. $\mathbf{S}^{5}$ can be described as a circle bundle over $\mathbb{C P}^{2}$, and the vector field is along the circle. At $u=0$ the $\mathbf{S}^{5}$ then shrinks to $\mathbb{C} \mathbf{P}^{2}$. This particular case will be discussed more extensively in the next section. For the general case, we can identify $\mathbb{R}^{6}$ with 
$\mathbb{C}^{3}$ and introduce the following coordinates

$$
\left(z_{1}, z_{2}, z_{3}\right)=\left(\frac{e^{i \alpha} r \cos \theta}{\sqrt{1+r^{2}}}, \frac{e^{i \beta} r \sin \theta}{\sqrt{1+r^{2}}}, \frac{e^{i \gamma}}{\sqrt{1+r^{2}}}\right)
$$

In these coordinates, the deformation of the sphere only affects the three coordinates $\alpha, \beta$ and $\gamma$. The vector $M \hat{n}$ is solely along this torus and, for generic ratios between these angles, the flow is dense in this torus. However, this is not a true fibration, and to avoid such complications we will only work with the simpler case.

\subsection{The Hopf Fibration}

The case when all three eigenvalues of $M$ are equal is the case where all of the scalar fields have the same dipole length. The analysis of the UV behavior of the theory will significantly simplify in this situation.

We set $\frac{1}{2} \tilde{L} \equiv \alpha_{1}=\alpha_{2}=\alpha_{3}$ and

$$
M=\left(\begin{array}{rrrrrr}
0 & -\tilde{L} & 0 & 0 & 0 & 0 \\
\tilde{L} & 0 & 0 & 0 & 0 & 0 \\
0 & 0 & 0 & -\tilde{L} & 0 & 0 \\
0 & 0 & \tilde{L} & 0 & 0 & 0 \\
0 & 0 & 0 & 0 & 0 & -\tilde{L} \\
0 & 0 & 0 & 0 & \tilde{L} & 0
\end{array}\right) .
$$

For $\tilde{L} \neq 0$, this choice of $M$ breaks all of the supersymmetry but it preserves a $U(3) \subset$ $S O(6)$ subgroup of the R-symmetry. The advantage of this choice of $M$ is that the factor $\hat{n}^{\top} M^{\top} M \hat{n}=\tilde{L}^{2}$ is independent of $\hat{n}$. According to the definition of $M$ in subsection (2.2), the bosons have dipole lengths $\pm 2 \pi \tilde{L}$, three of the fermions have dipole lengths $\pm \pi \tilde{L}$ and the remaining (complex) fermion has length $\pm 3 \pi \tilde{L}$.

We now write the metric on the deformed $\mathbf{S}^{5}$ explicitly. Let a unit vector $\hat{n}$ which parametrizes $\mathbf{S}^{5}$ in $\mathbb{C}^{3}$ be given by

$$
\hat{n}=\left(\frac{e^{i \gamma}}{\sqrt{1+|\alpha|^{2}+|\beta|^{2}}}, \frac{e^{i \gamma} \alpha}{\sqrt{1+|\alpha|^{2}+|\beta|^{2}}}, \frac{e^{i \gamma} \beta}{\sqrt{1+|\alpha|^{2}+|\beta|^{2}}}\right)
$$

with $\alpha$ and $\beta$ complex. Thus, the $\mathbf{S}^{5}$ is given as a circle fibration parametrized by $\gamma$ over $\mathbb{C P}^{2}$ parametrized by $\alpha$ and $\beta$. This is the famed Hopf fibration. The advantage of these 
coordinates is that the vector $M \hat{n}$ points along the direction of the fiber for $M$ given as in (6).

It can be shown that the metric on a regular $\mathbf{S}^{5}$ is in these coordinates

$$
d \hat{n}^{\top} d \hat{n}=\frac{|d \alpha|^{2}+|d \beta|^{2}}{1+|\alpha|^{2}+|\beta|^{2}}-\frac{|\bar{\alpha} d \alpha+\bar{\beta} d \beta|^{2}}{\left(1+|\alpha|^{2}+|\beta|^{2}\right)^{2}}+\left(d \gamma+\frac{\operatorname{Im}(\bar{\alpha} d \alpha+\bar{\beta} d \beta)}{1+|\alpha|^{2}+|\beta|^{2}}\right)^{2}
$$

where the first 2 terms describe the Fubini-Study metric on $\mathbb{C P}^{2}$.

For our deformed sphere, the metric is

$$
\begin{aligned}
d \hat{n}^{\top} d \hat{n}-\frac{\lambda^{2}\left(\hat{n}^{\top} M^{\top} d \hat{n}\right)^{2}}{u^{2}+\lambda^{2} \hat{n}^{\top} M^{\top} M \hat{n}}= & \frac{|d \alpha|^{2}+|d \beta|^{2}}{1+|\alpha|^{2}+|\beta|^{2}}-\frac{|\bar{\alpha} d \alpha+\bar{\beta} d \beta|^{2}}{\left(1+|\alpha|^{2}+|\beta|^{2}\right)^{2}} \\
& +\frac{u^{2}}{u^{2}+\lambda^{2} \tilde{L}^{2}}\left(d \gamma+\frac{\operatorname{Im}(\bar{\alpha} d \alpha+\bar{\beta} d \beta)}{1+|\alpha|^{2}+|\beta|^{2}}\right)^{2}
\end{aligned}
$$

The $5 \times 5$ determinant of the above metric can be calculated to be

$$
\operatorname{det} g=\left(\frac{u^{2}}{u^{2}+\lambda^{2} \tilde{L}^{2}}\right) \frac{1}{\left(1+|\alpha|^{2}+|\beta|^{2}\right)^{6}}
$$

Thus, the salient features of our deformed $\mathbf{S}^{5}$ are as follows

- It has the structure of an $\mathbf{S}^{1}$ (Hopf) fibration over a base $\mathbb{C P}^{2}$. An $S U(3)$ subgroup of $S O(6)$ acts freely on $\mathbb{C P}^{2}$.

- Invariance of the metric of the deformed $\mathbf{S}^{5}$ under $U(3) \subset S O(6)$ implies that the metric on the base $\mathbb{C} \mathbf{P}^{2}$ is independent of the position, and the metric on the fiber $\mathbf{S}^{1}$ is similarly homogeneous due to the $U(1)$ isometry which rotates the fibers.

- The radius of the fiber is independent of the $\mathbb{C P}^{2}$ coordinate and is given by

$$
\rho(u)=\mathcal{R} \frac{u}{\sqrt{u^{2}+\lambda^{2} \tilde{L}^{2}}} .
$$

- The volume of $\mathbb{C} \mathbf{P}^{2}$ is constant and given by

$$
\text { Volume }\left(\mathbb{C} \mathbf{P}^{2}\right)=\frac{\pi^{2}}{2} \mathcal{R}^{4}
$$

Finally, in these coordinates, the NSNS 2-form is given by

$$
B=-\frac{\lambda^{2} \tilde{L}}{u^{2}+\lambda^{2} \tilde{L}^{2}} d x_{3} \wedge \psi
$$


Here $\psi$ is the global angular 1-form of the Hopf fibration. In the notation of (7), it is given by

$$
\psi=d \gamma+\frac{\operatorname{Im}(\bar{\alpha} d \alpha+\bar{\beta} d \beta)}{1+|\alpha|^{2}+|\beta|^{2}}
$$

The 3-form field strength is given by

$$
H=d B=-\frac{\lambda^{2} \tilde{L}}{u^{2}+\lambda^{2} \tilde{L}^{2}} d x_{3} \wedge d \psi+\frac{\lambda^{2} \tilde{L} u}{\left(u^{2}+\lambda^{2} \tilde{L}^{2}\right)^{2}} d u \wedge d x_{3} \wedge \psi
$$

Here $d \psi$ is the closed harmonic 2-form that generates $H^{2}\left(\mathbb{C} \mathbf{P}^{2}, \mathbb{Z}\right)$.

\section{$7 \quad$ Nonlocality in the Supergravity Dual}

We now come to the heart of the paper. In this section, we will show how the nonlocality of the field theory is manifested in the geometry of the boundary of the supergravity. We will continue to work with the special case described above in (6.2). In this situation, as described in that section, the fiber shrinks to zero size on the boundary, and, as such, should be T-dualized to obtain a classical description. This will make the dipole nature of the nonlocality evident.

\subsection{T-duality of the Fiber}

As we approach the boundary of our solution, $u \rightarrow 0$, the volume of the base $\mathbb{C} \mathbf{P}^{2}$ remains a constant. However, the circle fibered along it shrinks to zero size. Note that the dilaton also approaches zero since

$$
e^{2\left(\phi-\phi_{0}\right)}=\frac{u^{2}}{u^{2}+\lambda^{2} \tilde{L}^{2}}
$$

It is easy to see that the curvature of the deformed $\mathbf{S}^{5}$ is still of the order of magnitude of $\frac{1}{\mathcal{R}^{2}}$, even when $u \ll \lambda \tilde{L}$. However, when $\rho(u)$ becomes of the order of magnitude of the string length, $\alpha^{1 / 2}$, we cannot trust the supergravity approximation anymore. This happens when $u \sim \alpha^{1 / 2} \mathcal{R}^{-1} \lambda \tilde{L}=\lambda^{1 / 2} \tilde{L}$.

Since the circle shrinks to zero, we have to perform T-duality on that direction. As we shall see in section (8), there is a subtlety that complicates matters, but for the time being we will naively apply the standard T-duality formulae. 
Again using the equations of 33, 34, 35, we obtain type-IIA with the metric

$$
\begin{gathered}
d s^{2}=\frac{\mathcal{R}^{2}}{u^{2}}\left(d t^{2}-d x_{1}^{2}-d x_{2}^{2}-d u^{2}\right)-\frac{\mathcal{R}^{2}}{u^{2}}\left(d x_{3}+\tilde{L} d \gamma\right)^{2}-\frac{\alpha^{\prime 2}}{\mathcal{R}^{2}} d \gamma^{2} \\
-\left(\text { constant } \mathbb{C P}^{2}\right) .
\end{gathered}
$$

We also have

$$
\begin{aligned}
e^{2 \phi} & =\frac{e^{2 \phi_{0}}}{\lambda}=\sqrt{\frac{g_{s}^{3}}{4 \pi N}} \\
\sum_{b} H_{u 3 b} d x^{b} & =-\frac{\lambda^{2} \tilde{L} u}{\left(u^{2}+\lambda^{2} \tilde{L}^{2}\right)^{2}} \frac{\operatorname{Im}(\bar{\alpha} d \alpha+\bar{\beta} d \beta)}{1+|\alpha|^{2}+|\beta|^{2}} .
\end{aligned}
$$

where $H$ is the 3 -form NSNS field strength. In addition, there is a non-trivial RR 4-form field strength which we will not write down. Note that the type-IIA dilaton becomes a constant. Despite the ominous factor $\frac{\alpha^{\prime 2}}{\mathcal{R}^{2}} \ll 1$ in (10), we see that type-IIA supergravity is a good approximation. No two points that are closer than $\alpha^{\prime 1 / 2}$ are identified. The only identification is

$$
\left(\ldots, x_{3}, \gamma\right) \sim\left(\ldots, x_{3}, \gamma+2 \pi\right)
$$

and the distance between those two points is large when $u \rightarrow 0$.

\subsection{Nonlocality on the Boundary}

The metric in equation (10) is a striking manifestation of the nonlocality of the field theory in the boundary metric. It describes the $x_{3}$ direction fibered over a small circle of radius $\frac{\alpha^{\prime}}{\mathcal{R}}$ parameterized by $\gamma$. The proper distance between the point with coordinates $\left(x_{3}, \gamma\right)$ and the point with coordinates $\left(x_{3}+2 \pi \tilde{L}, \gamma\right) \sim\left(x_{3}, \gamma-2 \pi\right)$ is $\frac{2 \pi \alpha^{\prime}}{\mathcal{R}}$ which is of stringy scale. On the other hand, the proper distance between $\left(x_{3}, \gamma\right)$ and $\left(x_{3}+\Delta, \gamma\right)$ is of order $\frac{\mathcal{R}}{u} \rightarrow \infty$ when $\Delta$ is not an integer multiple of $2 \pi \tilde{L}$ and $u \rightarrow 0$. In the field theory, this is translated into nonlocal interactions between fields at points that are separated by a distance of $L=2 \pi \tilde{L}$. If we think of the matter content of the dual SYM theory as constituting momentum modes along the $\mathbf{S}^{5}$, then, after T-duality, the nonlocality should be reflected in the winding number around the T-dual circle. This is exactly what we see here.

The metric (10) also shows that the $4 \mathrm{D}$ superconformal group is restored since the new coordinate $x_{3}+\tilde{L} \gamma$ can be attached to the $\mathbf{A d S}_{4}$ part of the metric to form $\mathbf{A d S}_{5}$. This 
is to be expected because the nonlocal interactions have a minimal distance $L$. At short distances the vicinity of each point should look like a 4D CFT and the interactions with fields at distance $L$ seems like an interaction with extra degrees of freedom outside the small neighborhood of the point.

\subsection{A Note on Momentum Conservation}

It is interesting to note that, because $\mathbf{S}^{5}$ is contractible, the winding number along the $\mathbf{S}^{1}$ fiber is not conserved. This is equivalent to the fact that the fibration has a nontrivial first Chern class. In order to contract the circle, however, one needs to pull it around a nontrivial 2-cycle of the base $\mathbb{C P}^{2}$. So, a concrete process that violates winding number conservation is to start with a small string on $\mathbb{C P}^{2}$ and then to gradually increase its size until it extends around the equator of a topologically nontrivial $\mathbb{C} \mathbf{P}^{1} \cong \mathbf{S}^{2}$ inside $\mathbb{C} \mathbf{P}^{2}$. Then we contract the string along the other hemisphere of the $\mathbb{C} \mathbf{P}^{1}$. At the end of the process, the string is wound around the fiber $\mathbf{S}^{1}$. This process requires energy scales of the order of the circumference of the equator of the $\mathbb{C P}^{1}$, i.e., $E \sim \mathcal{R} / \alpha^{\prime}$

Because of this, after T-duality, momentum along the $\gamma$-direction also must not be conserved. After T-duality, the $\gamma$-circle is fibered trivially over the $\mathbb{C P}^{2}$. Instead, we have a 3-form NSNS field strength, $H_{\gamma a b}$, along the circle and two directions inside the $\mathbb{C P}^{2}$. It is easy to see that $H_{\gamma a b}$ is proportional to $d \gamma \wedge \omega$ where $\omega$ is the harmonic 2 -form on $\mathbb{C P}^{2}$.

The process that violates momentum conservation along the $\gamma$-direction is the same as before. We start with a pointlike string inside $\mathbb{C P}^{2}$ and deform it to go around a nontrivial 2-cycle inside $\mathbb{C P}^{2}$ and then shrink it back to a point. Let $X(\sigma, \tau)$ be the closed path of the string as a function of time $\tau$ and string coordinate $0 \leq \sigma \leq 2 \pi$. Note that when both $\sigma$ and $\tau$ vary, the function $X(\sigma, \tau)$ spans a surface that is homologically equivalent to the nontrivial 2-cycle inside $\mathbb{C P}^{2}$. The violation of momentum conservation is due to the "magnetic" forces on a moving string in the presence of an $H=d B$ field strength. The total $\gamma$-momentum transfer is

$$
\int F_{\gamma}(\tau) d \tau=\int H_{\gamma a b} \partial_{\sigma} X^{a} \partial_{\tau} X^{b} d \sigma d \tau=\int \omega=1
$$

The RHS is the integral of the 2 -form $\omega$ along the nontrivial 2-cycle. 


\section{The Fermions}

In the previous section we saw that T-duality on the $\mathbf{S}^{1}$ fiber of the deformed $\mathbf{S}^{5}$ leads to a simple picture of nonlocality on the boundary. The nonlocality scale, $L=2 \pi \tilde{L}$, of the field theory matched nicely with the nonlocality scale on the boundary. In general, the proper distance between any two distinct points along the $x_{3}$-axis becomes infinite on the boundary because of the large rescaling factor $\frac{1}{u^{2}}$. If the $x_{3}$ coordinates of the two points differ by an integer multiple of $L$ then, as we saw in subsection (7.2), one can make a "shortcut" through an extra dimension that came from the T-dual of the $\mathbf{S}^{1}$ and go from one point to the other via a path whose proper length is shorter than the string scale.

However, the logic behind this picture is incomplete. To understand the problem, we will begin with a puzzle.

\subsection{What About the Fermions?}

The supergravity metric presents a nonlocal behavior that connects two points at $x_{3}$-distance of $L$, and this is indeed the dipole vector of the scalars of our field theory. But what about the fermions? Their dipole vectors, as mentioned below equation (6), are $\pm \frac{L}{2}$ or $\pm \frac{3 L}{2}$.

One might try to argue that we should only consider fermion bilinear operators but this does not appear to be the case. Obviously, there are fermionic operators in the theory. Moreover, let us consider gauge invariant operators in the field theory that also carry Rsymmetry charge. Specifically, let us consider the $U(1)_{c}$ center of the $U(3) \subset \operatorname{Spin}(6)_{R}$ that keeps the dipole matrix (6) invariant. This $U(1)_{c}$ acts on the $\mathbf{S}^{5}$, and it is easy to see that it is represented by rotations of the fiber $\mathbf{S}^{1}$. The dipole vector of any field $\Phi$ is given by $\frac{1}{2} L$ times its $U(1)_{c}$ charge. To make a gauge invariant operator we need to include an open Wilson line, for example

$$
W=\operatorname{tr}\left\{\Phi(x) e^{i \int_{C} A_{\mu} d x^{\mu}}\right\}
$$

where $C$ is an open path whose endpoints are at $x_{3}-\frac{L}{2}$ and $x_{3}+\frac{L}{2}$.

In the supergravity dual, closed Wilson lines correspond to closed paths on the boundary 42. The operator $W$ will also correspond to a closed path. It is the path that starts along $C$ on the boundary and then winds around the T-dual $\mathbf{S}^{1}$ to make the shortcut from $\left(x_{3}-\frac{L}{2}\right)$ to $\left(x_{3}+\frac{L}{2}\right)$. Note that after T-duality, the $U(1)_{c}$ charge is mapped to winding number along 
the T-dual $\mathbf{S}^{1}$. See also [57] for a related discussion.

Now suppose that $\Phi$ is a fermion dipole field of length $\frac{L}{2}$. There is no way to close the Wilson line in the supergravity dual.

\subsection{A Missing $(-)^{F}$}

The problem with the T-duality argument of subsection (7.1) is revealed by a careful analysis of the boundary conditions of the fermions around the fiber $\mathbf{S}^{1}$ of the Hopf fibration of $\mathbf{S}^{5}$. As we will now argue, the fermions have anti-periodic boundary conditions around the $\mathbf{S}^{1}$, and one should include $(-)^{F}$ in the boundary conditions, where $F$ is the fermion number. Our setting is reminiscent of the geometry in [43].

An observer living on our deformed $\mathbf{S}^{5}$ who cannot venture out over distances of the order of $\mathcal{R}$ sees only a small neighborhood $\mathcal{U}$ of $\mathbb{C P}^{2}$. In this neighborhood, fields vary slowly and the fiber $\mathbf{S}^{1}$ is noncontractible. The fibration has the structure of $\mathcal{U} \times \mathbf{S}^{1}$, and, if our local observers wish to describe fermions in their neighborhood, they have the option of choosing either periodic or anti-periodic boundary conditions around $\mathbf{S}^{1}$. The geometric holonomy around $\mathbf{S}^{1}$, calculated from the Levi-Civita connection, is the identity in $S O(5)$. However, as it turns out, the small $\mathbf{S}^{1}$ fiber is contractible inside the whole deformed $\mathbf{S}^{5}$, but in order to shrink it to a point one must first deform the circle to a path of length at least $2 \pi \mathcal{R}$. This fact allows one to calculate the holonomy for fermions around the fiber $\mathbf{S}^{1}$, and, as we will see below, it is $-1 \in \operatorname{Spin}(5)$. Thus a local observer would have to choose the anti-periodic boundary conditions and insert $(-)^{F}$ in every calculation.

In more mathematical terms, let $\mathbf{T}_{*} \mathbf{S}^{5}$ be the tangent bundle over $\mathbf{S}^{5}$. To define spinors on $\mathbf{S}^{5}$ we need the spin bundle, $\mathcal{S}$ over $\mathbf{S}^{5}$. The structure group of $\mathcal{S}$ is $\operatorname{Spin}(5)$ which is a double cover of $S O(5)$. Now pick a fiber $\mathbf{S}^{1}$ over a point $p$ of the base $\mathbb{C} \mathbf{P}^{2}$. Take a neigborhood $\mathcal{U} \in \mathbb{C} \mathbf{P}^{2}$ of the point $p$. The restriction of the $\mathbf{S}^{1}$-fibration to $\mathcal{U}$ is a manifold that is of the form $\mathcal{U} \times \mathbf{S}^{1}$. Over $\mathcal{U} \times \mathbf{S}^{1}$ there are two possible spin structures. In the first one, $\mathcal{S}_{+}$, the spinors have periodic boundary conditions around the $\mathbf{S}^{1}$ and in the second, $\mathcal{S}_{-}$, the spinors have anti periodic boundary conditions. The appropriate spin structure can be calculated from the $\operatorname{Spin}(5)$ holonomy around $\mathbf{S}^{1}$ in $\mathcal{S}$. It turns our that the holonomy is $-1 \in \operatorname{Spin}(5)$. To see this one can continuously deform the fiber $\mathbf{S}^{1}$ to a point inside $\mathbf{S}^{5}$ and 
trace the holonomy around the closed loop as it changes from $1 \in \operatorname{Spin}(5)$ when the loop is a point to $-1 \in \operatorname{Spin}(5)$ when the loop becomes the fiber. To actually calculate the holonomy, note that the fiber $\mathbf{S}^{1}$ is a circle of radius $\mathcal{R}$ inside $\mathbf{S}^{5}$. Pick an $\mathbf{S}^{2} \subset \mathbf{S}^{5}$ that contains $\mathbf{S}^{1}$ as its equator. Over $\mathbf{S}^{2}, \mathcal{S}$ reduces to the spin bundle of $\mathbf{S}^{2}$ times a trivial bundle. So the holonomy is the same as the holonomy of a spinor on $\mathbf{S}^{2}$ around the equator which is -1 .

It may seem at first sight that because of the $(-)^{F}$ we are actually describing strings at high temperature as in [44] (and see also [45]-46] for recent discussions). The closed string spectrum would then develop a tachyon when the $\mathbf{S}^{1}$ shrinks to a size smaller than the string scale, and our discussion would be rendered invalid. However, the same $-1 \in \operatorname{Spin}(5)$ holonomy is there even for the supersymmetric $\mathbf{A d} \mathbf{S}_{5} \times \mathbf{S}^{5}$ since the geometry of the $\mathbf{S}^{5}$ is the same except for the size of the fiber. This means that the $\mathbf{S}^{5}$ must support covariantly constant spinors, and something else should cancel the $-1 \in \operatorname{Spin}(5)$ phase. Indeed, the Dirac equation of motion for a fermion $\psi$ on $\mathbf{A d} \mathbf{S}_{5} \times \mathbf{S}^{5}$ contains an extra term, in addition to the spin connection. This term is proportional to $\left(F_{5}\right)_{\mu_{1} \ldots \mu_{5}} \Gamma^{\mu_{1} \ldots \mu_{5}} \psi$ where $F_{5}$ is the 5 -form RR field strength (see, for example, [47]). When integrated around the fiber $\mathbf{S}^{1}$, this extra term gives an additional phase of $(-1)$ so that altogether a covariantly constant spinor is possible for an $S O(6)$-symmetric $\mathbf{S}^{5}$.

It is important to point out that the $(-)^{F}$ phase coming from the geometric holonomy is a global effect. If we return to our local observer on $\mathbf{S}^{5}$, the $(-)^{F}$ rule will seem to them as an arbitrary rule of nature. On the other hand the $(-)^{F}$ phase coming from $F_{5}$ can be calculated locally. This has important implications to the application of T-duality.

\subsection{T-duality with $(-)^{F}$}

The anti-periodic boundary conditions for the fermions around the fiber $\mathbf{S}^{1}$ imply that we cannot just perform T-duality and get a type-IIA background with a large $\mathbf{S}^{1}$. Instead we get a type-0A theory. Such theories where discussed in 48]-56]. Their spectrum contains no fermions, and their bosonic massless spectrum is the same as that of type-IIA string theory but with two copies of every field in the RR sector. The main complication is that they also contain a tachyon. However, in our case the tachyon could very well be absent. In [44] the tachyon came from a string winding state in the RR sector. There, because of the extra $(-)^{F}$, there was a negative zero point energy for the worldsheet oscillators. In 
our case, as we have seen above, the $F_{5}$ term cancels the $(-)^{F}$, and therefore the winding state is quite likely to remain massive. The disappearance of the tachyonic instability is also supported by the arguments of [56]. There it was argued that a background RR flux provides a positive shift to the (mass) $)^{2}$ of the tachyon. If that is indeed the case, it is plausible that the UV region is described by type-0A string theory with the general features of the weakly curved metric described in section (7.1). Note that the magnitude of the 4-form RR field strength in type-0A is $\frac{M_{s}^{4}}{g_{s}}=g_{s}^{1 / 3} M_{p}^{4}$. This means that when $g_{s}$ is small, this field strength is large relative to the string scale but small relative to the Planck scale. Thus it appears that our dipole theory describes an RG flow from type-IIB string theory to type-OA string theory. However, quantizing strings in strong RR backgrounds remains an open problem, and type-0A is also likely to have a large cosmological constant, so this conjecture is hard to verify.

\subsection{Resolution of the Puzzle}

Assuming that T-duality to a weakly coupled type-0A theory is possible, the puzzle about spinor operators with half-integral dipole length is resolved as follows. The type-IIB compactification on $\mathbf{S}^{1}$ of radius $R$ with the extra $(-)^{F}$ twist can be described as the orbifold of a compactification on a circle of radius $2 R$ by the $\mathbb{Z}_{2}$ action $(-)^{F+P}$ where $P$ is the KaluzaKlein momentum. The T-dual is therefore an orbifold of type-IIA on a circle of radius $\frac{1}{2 R}$ by $(-)^{F+W}$ where $W$ is the winding number. Now we see that in the untwisted sector of the T-dual background, strings that are spacetime bosons must have even winding number, and strings that are spacetime fermions must have odd winding number. Thus, the resolution of the puzzle is that the dual theory is not type-IIA on a circle of radius $\frac{1}{R}$ but rather type-0A on a circle of radius $\frac{1}{2 R}$.

\section{Correlation Functions}

In a local field theory, correlation functions of operators, $\langle O(x) O(y)\rangle$, have short distance singularities when $x \rightarrow y$. In dipole theories, we expect a singularity to appear also when $x \rightarrow y \pm L_{i}$, where $L_{i}$ is one of the characteristic vectors of nonlocality as in section (2).

\footnotetext{
${ }^{1}$ We are grateful to Igor Klebanov for pointing this out.
} 
In the special case we study in this paper, the length of the characteristic vectors of the scalars is $L=2 \pi \tilde{L}$. For operators $O(x)$ that have no dipole length of their own (for example $\left.\operatorname{tr}\left\{F_{\mu \nu}^{2}\right\}\right)$ we therefore expect

$$
\langle O(x) O(y)\rangle \rightarrow_{x \rightarrow y+L} \frac{C}{|x-y-L|^{2 \Delta}}
$$

and then in momentum space we expect to find a term that behaves like

$$
\left\langle O(k)^{\dagger} O(k)\right\rangle \longrightarrow_{k \rightarrow \infty} \frac{C e^{i k \cdot L}}{k^{4-2 \Delta}} .
$$

For operators $O(x)$ that do have a length we expect the behavior of the correlation function to be more complicated since the operators contain nonlocal Wilson lines as in (11). It is likely that the correlation functions exhibit an exponential behavior $\sim e^{\sqrt{(\text { const })\left|k_{3}\right| L}}$ analogous to that of noncommutative geometry [57, 58]. 2] For the rest of this discussion we will restrict ourselves to operators $O(x)$ with dipole length zero.

We can use the AdS/CFT correspondence to compute these correlation functions in the large $N$ limit. We will restrict ourselves to the special case where the R-symmetry is broken from $\operatorname{Spin}(6)$ down to $U(3)$, as in section (6.2). Because the AdS/CFT correspondence directly probes the nonperturbative nature of the field theory, it is perhaps a bit too much to expect to see the exact form above, but, in the limit of high momentum along the dipole direction, a sign of nonlocality would be a rapid oscillation in the correlation function in momentum space.

It is, in general, a difficult problem to decouple the fields on a nontrivial background such as any of the examples in this paper. Following [57], we will simply postulate that there exists a massless scalar living on our spacetime. In particular, it should satisfy the field equation

$$
\partial_{\mu}\left(e^{-2 \phi} \sqrt{\operatorname{det} g} g^{\mu \nu} \partial_{\nu} \Phi(\vec{x}, u)\right)=0
$$

where $\vec{x}=\left(t, x_{1}, x_{2}, x_{3}\right)$.

This is still quite a difficult problem to solve, but we will soon see how it can be simplified. In particular, we recall the determinant of the metric of the sphere, (8), in the Hopf fibration coordinates. Including the AdS portion of the metric, we have

$$
\operatorname{det} g=\mathcal{R}^{20}\left(\frac{u^{2}}{u^{2}+\lambda^{2} \tilde{L}^{2}}\right)^{2} u^{-10} \frac{1}{\left(1+|\alpha|^{2}+|\beta|^{2}\right)^{6}}
$$

\footnotetext{
${ }^{2}$ We are grateful to M. Rozali for a discussion on this point.

${ }^{3}$ We are grateful to I.R. Klebanov for explaining the relevant issues to us.
} 
We immediately see that this factors into a contribution that depends on the sphere and one that depends on the AdS. Thus, because our metric is block diagonal, we can choose our scalar field to be constant on the sphere, and all contributions from the sphere will cancel out of our equations. Another happy fact is that the contribution from the dilaton exactly cancels the $u^{2} /\left(u^{2}+\lambda^{2} \tilde{L}^{2}\right)$ term reducing this to almost the standard massless field equation on AdS space.

As usual, the most interesting part of the equation comes from the $u$ coordinate, so we write

$$
\Phi(\vec{x}, u)=\varphi(u) e^{i \vec{k} \cdot \vec{x}}
$$

Then $\varphi$ satisfies the following equation

$$
u^{3} \partial_{u}\left(\frac{1}{u^{3}} \partial_{u} \varphi(u)\right)+\left(k^{2}-\frac{\left(\lambda k_{3} \tilde{L}\right)^{2}}{u^{2}}\right) \varphi(u)=0
$$

If we expand this, we obtain

$$
\varphi^{\prime \prime}-\frac{3}{u} \varphi^{\prime}+\left(k^{2}-\frac{\left(\lambda k_{3} \tilde{L}\right)^{2}}{u^{2}}\right) \varphi=0 .
$$

We recognize this as the equation for a massive field in ordinary $\mathbf{A d S}$ space with $m \mathcal{R}=\lambda k_{3} \tilde{L}$. Thus, we can copy the final result from equation (44) of [2]

$$
\langle\mathcal{O}(k) \mathcal{O}(q)\rangle=-(2 \pi)^{4} \delta^{4}(k+q) \frac{N^{2}}{8 \pi^{2}} \frac{\Gamma(1-\nu)}{\Gamma(\nu)}\left(\frac{k \mathcal{R}}{2}\right)^{2 \nu} \mathcal{R}^{-4}
$$

where $\nu=\sqrt{4+\left(k_{3} \tilde{L}\right)^{2}}$.

Let us now take the limit that $k_{3} \rightarrow \infty$. In this limit, we have

$$
\langle\mathcal{O}(k) \mathcal{O}(-k)\rangle \sim \frac{1}{\sin (\pi \nu)}\left(\frac{(k \mathcal{R} / 2)^{\nu}}{\Gamma(\nu)}\right)^{2} \stackrel{\lambda{ }_{3} \rightarrow \infty}{\sim} \frac{\lambda k_{3} \tilde{L}\left(\frac{|k| e \mathcal{R}}{2 \lambda k_{3} \tilde{L}}\right)^{2 \lambda k_{3} \tilde{L}}}{\sin \left(\pi \lambda k_{3} \tilde{L}\right)}
$$

It exhibits an oscillatory behavior but not quite what we have anticipated. We expected the wavelength of the nonlocal behavior to be an integer multiple of the dipole length. This is not what we observe here. This is a puzzling phenomenon, but it is consistent with the observation from the supergravity dual that the scale of the nonlocality is actually $\lambda \tilde{L}$ rather than just $\tilde{L}$. Since $\lambda \tilde{L}>>\tilde{L}$ there is no immediate contradiction. It could be that in the large $\lambda$ limit the dominant contribution to the nonlocal behavior of the correlation function 
comes from the nonlocality on scale $[\lambda] \tilde{L}$ (where $[\lambda]$ is the integer that is closest to $\lambda$ ). It is important to realize, however, that the supergravity approximation ceases to be valid when $u<\alpha^{1 / 2} \mathcal{R}^{-1}$, as we explained in subsection (7.1). This suggests that the above calculation may not be entirely valid. This is worthy of further investigation.

\section{Discussion}

In this paper, we have shown how the nonlocality of dipole theories is manifested in the supergravity dual. We discovered that the metric becomes degenerate at the boundary of the spacetime and that this could be used to explicitly demonstrate the nonlocality. Although this feature of the metric was shown using the naive T-duality to type-IIA and, as we argued in section (8), one actually gets type-0A with a strong RR field strength, we believe that the metric still has this general structure. This should be a generic feature of the supergravity duals of nonlocal field theories. It is not a surprising result. Nonlocality, when realized in some limit of string theory, cannot be a purely supergravity effect. The nonlocality must be a result of the inclusion of some stringy degrees of freedom on the boundary. The degeneracy of the metric in string frame means that we cannot treat the boundary as classical, and this is the source of the nonlocality.

It is worthwhile to compare this situation to that in noncommutative geometry to see if we can distill some more general features of the supergravity dual. The discussion that follows has some features in common with [31, [59]-[62]. H]

Recall that the metric of the supergravity dual of NCSYM is [5, 6] (ignoring dimensionless constants)

$$
d s^{2}=\frac{1}{u^{2}}\left(d t^{2}-d x_{1}^{2}-\frac{u^{4}}{u^{4}+\theta^{2}}\left(d x_{2}^{2}+d x_{3}^{2}\right)-d u^{2}\right)
$$

The other fields are

$$
\begin{aligned}
e^{2 \phi} & =\frac{u^{4}}{u^{4}+\theta^{2}} \\
B_{23} & =-\frac{\theta}{u^{4}+\theta^{2}}
\end{aligned}
$$

\footnotetext{
${ }^{4}$ We are grateful to A. Hashimoto for pointing out some of these references and for discussing this with us.
} 
We see that both the second and third directions go to zero length on the boundary, indicating some sort of stringy effect. Note that here, the degeneracy is in the AdS part of the metric indicating that the nonlocality is part of the space that the field theory lives on. This is in contrast to our dipole theories where the degeneracy is on the $\mathbf{S}^{5}$ indicating that the nonlocality is part of the field content of the theory.

Following the same procedure as in (7.2), we compactify these directions and T-dualize along one of them, say the second. As before, the presence of the B-field gives rise to cross terms in the metric. Specifically, after T-duality, we have, isolating the 2 and 3 directions

$$
d s^{2}=\left(u^{4}+\theta^{2}\right) \frac{d x_{2}^{2}}{u^{2}}+2 \theta \frac{d x_{2} d x_{3}}{u^{2}}+\frac{u^{2}+\theta^{2} u^{-2}}{u^{4}+\theta^{2}} d x_{3}^{2}
$$

If we take the $u \rightarrow 0$ limit, we can rewrite this as

$$
d s^{2}=\frac{1}{u^{2}}\left(\theta d x_{2}+d x_{3}\right)^{2}
$$

This has almost the same form as the metric we obtained in section (7.1). When we traverse the 2-circle, the above coordinate gets shifted by $\theta$. As T-duality interchanges momentum with winding, we interpret this as a dipole in the 3 direction with length equal to $\theta$ times the momentum. This is exactly the situation in NCSYM.

What are the general features of the supergravity duals of nonlocal field theories that we can infer from this?

- The metric becomes degenerate on the boundary of AdS.

- The NSNS 2-form field has a component along the degenerate direction.

- We can (perhaps after compactification) T-dualize along this direction.

- After T-duality, the NSNS 2-form field induces off-diagonal terms in the metric that can be interpreted as a fibration over a string scale circle.

- The nonlocality of the field theory is manifested by the shift in the new coordinate as we go around the string scale circle.

While these features may not be generic for all nonlocal theories, it is not unreasonable to assume that they may be generic for the generalized dipole theories mentioned at the end of section (2.1) of which both the dipoles discussed here and those of noncommutative 
geometry are a special case. In [10] a generalization of dipoles to the case of the $(2,0)$ theory was proposed where, instead of constant length dipoles, there are constant area "discpoles". This should have a supergravity dual of the from $\mathbf{A d S}_{7} \times \mathbf{S}^{4}$. It would be interesting to investigate the effects of nonlocality on the supergravity in this situation.

Before concluding let us return to a loose end from the beginning of section (3). We mentioned that the twisted string theory backgrounds are unstable if supersymmetry is broken. This instability was discussed in [24, 25, 26] and is related to the instability of Kaluza-Klein compactifications without supersymmetry [27. In section (6.2) we used a nonsupersymmetric twisted theory, and we therefore expect it to be unstable. However, the probability for decay per unit time and volume is exponentially suppressed as $g_{s} \rightarrow 0$. In the large $N$ limit (keeping $g_{s} N$ fixed) we can therefore assume that the background is stable. It is interesting to ask whether the dipole field theory on the probe is also unstable. We will not address this question here. One possibility suggested by $\mathrm{O}$. Aharony is that a potential is generated on the Coulomb branch of the dipole field theory that makes the origin unstable. This is currently under investigation.

\section{Acknowledgments}

We are grateful to O. Aharony, J. Gomis, R. Gopakumar, A. Hashimoto, C.P. Herzog, N. Itzhaki, I.R. Klebanov, J. Maldacena, D. Minic, S. Minwalla, B. Morariu, A. Polychronakos, N. Seiberg, S. Shatashvili, S. Shenker, A. Strominger, H. Verlinde, E. Witten and Z. Yin for helpful discussions and comments. KD would also like to thank the string theory group at Harvard University, where part of this work was done, for stimulating discussions. The research of OJG is supported by NSF grant no. PHY-9802484; the research of JLK is in part supported by the Natural Sciences and Engineering Research Council of Canada; the research of KD is supported by Department of Energy grant no. DE-FG02-90ER40542 and the research of GR is supported by NSF grant no. PHY-0070928 and by a Helen and Martin Chooljian fellowship. 


\section{A Generic Orientation of the Dipole Vectors}

In section (14) we promised to describe the supergravity solution for generic dipole theories where the various dipole vectors are not all along the same direction. In order to avoid clutter, we will set $\alpha^{\prime}=1$ in this appendix.

We start with a D3-brane extended in the 0123 directions, compactified on a $T^{3}$ with radii $\left(R_{1}, R_{2}, R_{3}\right)$. The relevant non-zero fields are

$$
\begin{aligned}
d s^{2} & =\frac{1}{\sqrt{H}}\left(d t^{2}-\left(R_{1} d x^{1}\right)^{2}-\left(R_{2} d x^{2}\right)^{2}-\left(R_{3} d x^{3}\right)^{2}\right)-\sqrt{H}\left(d x^{a}\right)^{2} \\
C_{0123} & =-\frac{1}{H} \\
\varphi & =\varphi_{0}
\end{aligned}
$$

where

$$
H=1+\frac{\mathcal{R}^{4}}{r^{4}} \quad r^{2} \equiv\left(x^{a}\right)^{2}
$$

The roman indices $a, b, \ldots$ run from 4 to 9 , and we use greek indices to indicate the compactified directions 1,2,3. Starting from the solution (12), we perform the T-duality transformation three times, in the three compactified directions using the formulae of [33, 34, 35]. The answer, which is a D0-brane smeared over the T-dual torus $T^{3}:\left(R_{1}^{-1}, R_{2}^{-1}, R_{3}^{-1}\right)$, is

$$
\begin{aligned}
d s^{2} & =\frac{1}{\sqrt{H}} d t^{2}-\sqrt{H}\left(\left(\frac{d x^{1}}{R_{1}}\right)^{2}+\left(\frac{d x^{2}}{R_{2}}\right)^{2}+\left(\frac{d x^{3}}{R_{3}}\right)^{2}\right)-\sqrt{H}\left(d x^{a}\right)^{2} \\
C_{0}^{(1)} & =-\frac{4}{H} \\
e^{2\left(\phi-\varphi_{0}\right)} & =\frac{H^{3 / 2}}{R_{1}^{2} R_{2}^{2} R_{3}^{2}}
\end{aligned}
$$

Now, we introduce the three twists, by replacing

$$
d x^{a} \longrightarrow d x^{a}-\sum_{\mu}\left(\Omega_{a b}^{\mu} x^{b}\right) d x^{\mu}
$$

where $\left(\Omega^{\mu}\right)^{\top}=-\Omega^{\mu}$ are commuting elements of $\mathrm{SO}(6)$. The metric with the twist is

$$
d s^{2}=\frac{1}{\sqrt{H}} d t^{2}-\sqrt{H}\left(\left(\frac{d x^{1}}{R_{1}}\right)^{2}+\left(\frac{d x^{2}}{R_{2}}\right)^{2}+\left(\frac{d x^{3}}{R_{3}}\right)^{2}\right)-\sqrt{H}\left(d x^{a}-\left(\Omega_{a b}^{\mu} x^{b}\right) d x^{\mu}\right)^{2}
$$

Now, we T-dualize three times to get back the metric for a D3-brane with a dipole theory living on it. Define $M^{\mu} \equiv R_{\mu} \Omega^{\mu}$ (no contraction over $\mu$ ) and $x^{a} \equiv r \hat{n}^{a}$ where $\hat{n}^{\top} \hat{n}=1$. With 
some work, the metric turns out to be (here and below there is no contraction in terms like $\left.R_{\nu} d x^{\nu}\right)$

$$
\begin{aligned}
d s^{2} & =\frac{1}{\sqrt{H}} d t^{2}-\sqrt{H} d r^{2} \\
& -\frac{1}{\sqrt{H}} \frac{\epsilon_{\alpha \beta \gamma} \epsilon_{\kappa \mu \nu}\left[\delta^{\alpha \kappa}+r^{2}\left(m^{\alpha}\right)^{\top} m^{\kappa}\right]\left[\delta^{\beta \mu}+r^{2}\left(m^{\beta}\right)^{\top} m^{\mu}\right]}{2 D}\left(R_{\gamma} d x^{\gamma}\right)\left(R_{\nu} d x^{\nu}\right) \\
& -\sqrt{H}\left(r^{2} d n^{T} d n\right) \\
& +\sqrt{H}\left(\frac{r^{4} \epsilon_{\alpha \beta \gamma} \epsilon_{\kappa \mu \nu}\left[\delta^{\alpha \kappa}+r^{2}\left(m^{\alpha}\right)^{\top} m^{\kappa}\right]\left[\delta^{\beta \mu}+r^{2}\left(m^{\beta}\right)^{\top} m^{\mu}\right]\left[\left(\left(m^{\gamma}\right)^{\top} d n\right)\left(\left(m^{\nu}\right)^{\top} d n\right)\right]}{2 D}\right)
\end{aligned}
$$

where we have defined

$$
D \equiv \frac{1}{6} \epsilon_{\alpha \beta \gamma} \epsilon_{\kappa \mu \nu}\left[\delta^{\alpha \kappa}+r^{2}\left(m^{\alpha}\right)^{\top} m^{\kappa}\right]\left[\delta^{\beta \mu}+r^{2}\left(m^{\beta}\right)^{\top} m^{\mu}\right]\left[\delta^{\gamma \nu}+r^{2}\left(m^{\gamma}\right)^{\top} m^{\nu}\right]
$$

and

$$
m^{\alpha} \equiv M^{\alpha} \hat{n}
$$

The other nonzero fields are

$$
\begin{aligned}
C_{0123}^{(4)} & =\frac{1}{H} \\
B_{\mu a}^{(1)} d x^{\mu} \wedge d \hat{n}^{a} & =-\sqrt{H} j_{\gamma \nu} d x^{\gamma} \wedge \frac{r m^{\mu} d \hat{n}}{R_{\nu}} \\
& =\frac{r \epsilon_{\alpha \beta \gamma} \epsilon_{\kappa \mu \nu}\left[\delta^{\alpha \kappa}+r^{2}\left(m^{\alpha}\right)^{\top} m^{\kappa}\right]\left[\delta^{\beta \mu}+r^{2}\left(m^{\beta}\right)^{\top} m^{\mu}\right]\left[\left(R_{\gamma} d x^{\gamma}\right) \wedge\left(\left(m^{\nu}\right)^{\top} d \hat{n}\right)\right]}{2 D} \\
e^{2\left(\varphi-\varphi_{0}\right)} & =\frac{1}{D}
\end{aligned}
$$

For $M^{1}=M^{2}=0$ this reduces to the answers for a single twist. It is interesting to ask what happens when the twists, $M^{\mu}$, do not commute. In this situation, the Ricci scalar of the twisted metric (14) has a field strength term, and thus the metric is no longer a solution to the supergravity equations.

\section{References}

[1] J. Maldacena, "The Large N Limit of Superconformal Field Theories and Supergravity," Adv. Theor. Math. Phys. 2 (1998) 231-252 [hep-th/9711200].

[2] S. S. Gubser, I. R. Klebanov and A. M. Polyakov, "Gauge theory correlators from non-critical string theory," Phys. Lett. B 428, 105 (1998) [hep-th/9802109]. 
[3] E. Witten, "Anti-de Sitter space and holography," Adv. Theor. Math. Phys. 2, 253 (1998) [hep-th/9802150].

[4] O. Aharony, S. S. Gubser, J. Maldacena, H. Ooguri and Y. Oz, "Large N field theories, string theory and gravity," Phys. Rept. 323, 183 (2000) [hep-th/9905111].

[5] A. Hashimoto and N. Itzhaki, "Non-commutative Yang-Mills and the AdS/CFT correspondence," Phys. Lett. B465, 142 (1999) [hep-th/9907166].

[6] J. M. Maldacena and J. G. Russo, "Large $N$ limit of non-commutative gauge theories," JHEP9909, 025 (1999) [hep-th/9908134].

[7] M. Alishahiha, Y. Oz and M. M. Sheikh-Jabbari, "Supergravity and large $N$ noncommutative field theories," JHEP9911, 007 (1999) [hep-th/9909215].

[8] S. Minwalla, M. Van Raamsdonk and N. Seiberg, "Noncommutative perturbative dynamics," JHEP0002, 020 (2000) [hep-th/9912072].

[9] M. Van Raamsdonk and N. Seiberg, "Comments on noncommutative perturbative dynamics," JHEP0003, 035 (2000) [hep-th/0002186].

[10] A. Bergman and O. J. Ganor, "Dipoles, twists and noncommutative gauge theory," JHEP0010, 018 (2000) [hep-th/0008030].

[11] K. Dasgupta, O.J. Ganor and G. Rajesh, "Vector Deformations of N=4 Super-YangMills Theory, Pinned Branes, and Arched Strings," hep-th/0010072

[12] E. Witten, "New 'gauge' theories in six dimensions," JHEP9801, 001 (1998) [hep-th/9710065].

[13] Y.-K.E. Cheung, O.J. Ganor and M. Krogh, "On the Twisted $(2,0)$ and Little String Theories," Nucl. Phys. B536 (1998) 175, [hep-th/9805045].

[14] Y.-K.E. Cheung, O.J. Ganor, M. Krogh and A.Yu. Mikhailov, "Noncommutative Instantons and Twisted (2,0) and Little String Theories," Nucl. Phys. B564 (2000) 259, [hep-th/9812172].

[15] N. Seiberg and E. Witten, "String Theory and Noncommutative Geometry," JHEP 9909, 032 (1999) [hep-th/9908142].

[16] M. Kreuzer and J. Zhou, "Lambda-symmetry and background independence of noncommutative gauge theory on $R^{n}$," JHEP0001, 011 (2000) [hep-th/9912174]. 
[17] O. J. Ganor, G. Rajesh and S. Sethi, "Duality and non-commutative gauge theory," Phys. Rev. D 62, 125008 (2000) [hep-th/0005046].

[18] D. Bigatti and L. Susskind, "Magnetic fields, branes and noncommutative geometry," Phys. Rev. D 62, 066004 (2000) [hep-th/9908056].

[19] Z. Yin, "A note on space noncommutativity," Phys. Lett. B 466, 234 (1999) [hep-th/9908152].

[20] M. M. Sheikh-Jabbari, "Open strings in a B-field background as electric dipoles," Phys. Lett. B 455, 129 (1999) [hep-th/9901080].

[21] J.G. Russo and A. Tseytlin, "Magnetic flux tube models in superstring theory," Nucl. Phys. B461 (1996) 131, [hep-th/9508068].

[22] J. de Boer and S. L. Shatashvili, "Two-dimensional conformal field theories on AdS(2d+1) backgrounds," JHEP9906, 013 (1999) [hep-th/9905032].

[23] M. A. Melvin, Phys. Lett. 8 (1964) 65.

[24] F. Dowker, J. Gauntlett, G. Gibbons and G. Horowitz, "The Decay of Magnetic Fields in Kaluza-Klein Theory," Phys. Rev. D52 6929-6940 (1995), [hep-th/9507143].

[25] M. Gutperle and M. Costa, "The Kaluza-Klein Melvin Solution in M-theory," JHEP0103, 027 (2001) [hep-th/0012072].

[26] A. Strominger, Talk at DavidFest, Santa-Barbara 2001.

[27] E. Witten, "Instability Of The Kaluza-Klein Vacuum", Nucl. Phys. B195 (1982) 481.

[28] Y. E. Cheung and M. Krogh, "Noncommutative geometry from 0-branes in a background B-field," Nucl. Phys. B 528, 185 (1998) [hep-th/9803031].

[29] M. M. Sheikh-Jabbari, "Super Yang-Mills theory on noncommutative torus from open strings interactions," Phys. Lett. B 450, 119 (1999) [hep-th/9810179].

[30] C. Chu and P. Ho, "Constrained quantization of open string in background $B$ field and noncommutative D-brane," Nucl. Phys. B 568, 447 (2000) [hep-th/9906192].

[31] M.R. Douglas and C. Hull, "D-branes and the Noncommutative Torus," JHEP. 9802 (1998) 008 [hep-th/9711165].

[32] M.R. Douglas and G. Moore, "D-branes, Quivers, and ALE Instantons," hep-th/9603167 
[33] T. Buscher, "A Symmetry of the String Background Field Equations," Phys. Lett. B 194, 59 (1987).

[34] T. Buscher, "Path Integral Derivation of Quantum Duality in Nonlinear Sigma Model," Phys. Lett. B 201, 466 (1988).

[35] E. Bergshoeff, C. Hull and T. Ortin, "Duality in the type II superstring effective action," Nucl. Phys. B 451, 547 (1995) [hep-th/9504081].

[36] S.S. Gubser, I.R. Klebanov and A.A. Tseytlin, "String Theory and Classical Absorption by Threebranes," Nucl. Phys. B 499, 217 (1997) [hep-th/9703040].

[37] S.S. Gubser and I.R. Klebanov, "Absorption by Branes and Schwinger Terms in the World Volume Theory," Phys. Lett. B413, 41 (1997) [hep-th/9708005].

[38] M. Alishahiha, H. Ita and Y. Oz, "Graviton Scattering on D6 Branes with B Fields," JHEP. 006 (2000) 002, [hep-th/0004011].

[39] H. J. Kim, L. J. Romans and P. van Nieuwenhuizen, "The Mass Spectrum Of Chiral $N=2 D=10$ Supergravity On $S^{5}, "$ Phys. Rev. D32 (1985) 32.

[40] K. Intriligator, "Bonus Symmetries of $\mathcal{N}=4$ Super-Yang-Mills Correlation Functions via AdS Duality," Nucl. Phys. B 551, 575 (1999)[hep-th/9811047].

[41] D. Z. Freedman, S. S. Gubser, K. Pilch and N. P. Warner, "Continuous distributions of D3-branes and gauged supergravity," JHEP0007, 038 (2000), [hep-th/9906194].

[42] J. Maldacena, "Wilson loops in large N field theories," Phys. Rev. Lett. 80, 4859 (1998) [hep-th/9803002].

[43] E. Witten, "Anti-de Sitter space, thermal phase transition, and confinement in gauge theories," Adv. Theor. Math. Phys. 2, 505 (1998) [hep-th/9803131].

[44] J. Atick and E. Witten, Nucl. Phys. B 310, 291 (1988).

[45] S. S. Gubser, S. Gukov, I. R. Klebanov, M. Rangamani and E. Witten, "The Hagedorn transition in non-commutative open string theory," [hep-th/0009140].

[46] S. A. Abel, J. L. Barbon, I. I. Kogan and E. Rabinovici, "Some thermodynamical aspects of string theory," [hep-th/9911004].

[47] I. R. Klebanov and E. Witten, "Superconformal field theory on threebranes at a CalabiYau singularity," Nucl. Phys. B 536, 199 (1998) [hep-th/9807080]. 
[48] L. Dixon and J. Harvey, "String theories in ten dimensions without space-time supersymmetry," Nucl. Phys. B 274, 93 (1986).

[49] N. Seiberg and E. Witten, "Spin structures in string theory," Nucl. Phys. B 276, 272 (1986).

[50] C. Thorn, unpublished.

[51] M. Bianchi and A. Sagnotti, "On the systematics of Open String Theories," Phys. Lett. B 247, 517 (1990).

[52] A. Sagnotti, "Some Properties of Open String Theories," hep-th/9509080

[53] A. Sagnotti, "Surprises in Open String Perturbation Theory," Nucl. Phys. Proc. Suppl. B 56, 332 (1997) [hep-th/9702093].

[54] C. Angelantonj, "Nontachyonic Open Descendants of the 0B String Theory," [hep-th/9810214].

[55] O. Bergman and M. Gaberdiel, "A Nonsupersymmetric Open String Theory and Sduality," Nucl. Phys. B 499, 183 (1997) [hep-th/9701137].

[56] I. R. Klebanov and A. A. Tseytlin, "D-branes and dual gauge theories in type 0 strings," Nucl. Phys. B 546, 155 (1999) [hep-th/9811035].

[57] D. J. Gross, A. Hashimoto and N. Itzhaki, "Observables of non-commutative gauge theories," hep-th/0008075

[58] M. Rozali and M. Van Raamsdonk, "Gauge invariant correlators in non-commutative gauge theory," hep-th/0012065.

[59] A. Hashimoto and N. Itzhaki, "On the hierarchy between non-commutative and ordinary supersymmetric Yang-Mills," JHEP9912, 007 (1999) [hep-th/9911057].

[60] N. Ishibashi, S. Iso, H. Kawai and Y. Kitazawa, "Wilson loops in noncommutative Yang-Mills," Nucl. Phys. B 573, 573 (2000) [hep-th/9910004].

[61] J. Ambjorn, Y. M. Makeenko, J. Nishimura and R. J. Szabo, "Lattice gauge fields and discrete noncommutative Yang-Mills theory," JHEP0005, 023 (2000) [hep-th/0004147].

[62] K. Saraikin, "Comments on the Morita equivalence," J. Exp. Theor. Phys. 91, 653 (2000) [hep-th/0005138]. 